\title{
Kikuchi-Fujimoto Disease: A Case Report and Review of the Literature
}

\author{
MMB Zaman ${ }^{1}$
}

\begin{abstract}
:
Kikuchi-Fujimoto disease is a rare benign, condition of necrotizing histiocytic lymphadenitis. Presenting complaints of neck masses in association with non-specific systemic signs and symptoms prompt investigation towards the more common diagnoses. However, rarer conditions must still be considered especially when a patient's condition fails to abate. Herein I discuss a case of female patient who presented with a neck mass that was not attributable to the more common causes. A 28 year old lady presented with feverish feeling, weight loss and tender cervical lymph nodes. Initially tuberculous lymphadenitis was suspected but Kikuchi-Fujimoto disease was diagnosed after cervical lymph node biopsy. Symptomatic treatment was provided and an eventful full recovery was made.
\end{abstract}

Key words: Kikuchi-Fujimoto, Necrotizing lymphadenitis.

\section{Case History:}

A 28 year old lady presented with a four weeks history of fever, anorexia and weight loss. She had no other complaints. She was previously fit and well. On examination she was a bit lethargic, but otherwise looked well. She was afebrile and haemodynamically stable. Significant finding was lymphadenopathy of left posterior cervical region. Lymph node was firm, tender and discrete. There was no overlying erythema, ulceration or sinus formation. There was no organomegaly. Blood tests revealed neutropenia, ESR was $48 \mathrm{~mm}$ in $1^{\text {st }}$ hour. Liver and renal function were normal. Montoux test was $06 \mathrm{~mm}$ on $3^{\text {rd }}$ day. Chest radiograph and abdominal USG were normal. FNAC was done but showed nonspecific lymphadenitis. Excisional biopsy of cervical lymph node was done and showed loss of nodal architecture with large area of necrosis with devoid of polymorphs and confirmed a diagnosis of Kikuchi-Fujimoto disease (KFD). Treatment was given with Ibuprofen 400mg 12 hourly for one month in combination with prednisolone $1 \mathrm{mg}$ $/ \mathrm{kg} /$ day for one month then tapering was done over next two months. On first follow up visit at one month she feels better, fever subsided, appetite improved and she also gained weight by two $\mathrm{kg}$. On Second follow up at third month of therapy she fully recovered and there was no residual lymphadenopathy.

1. Dr. M. M. Bodiuzzaman, MBBS, FCPS (Medicine), Junior consultant, Dept. of Medicine, Faridpur Medical College Hospital.

Address of correspondence :

Dr. M. M. Bodiuzzaman, MBBS, FCPS (Medicine), Junior consultant, Dept. of Medicine, Faridpur Medical College Hospital.

Phone-+88-0

E-mail :

\section{Discussion:}

Kikuchi-Fujimoto Disease (KFD) is a benign histiocytic necrotising lymphadenitis. It is rare, but most common in Asia. In the early 1970s both Kikuchi and Fujimoto first described cases of KFD in Japan ${ }^{1,2}$. KFD frequently found in East Asian countries, is rare in the UK. Its aetiology has not yet been fully determined, however it is believed that it may be of viral origin, EBV, HHV6 and 8 have been suggested. Raw fish was postulated as a cause, but the recent literature doesn't support this ${ }^{3}$. An autoimmune aetiology is also likely as it has been reported in association with SLE. It tends to affect a young population under 30 years of age, including children, although the latter are less commonly affected. There are reported cases in an older age group and pregnant women too ${ }^{4}$. Early reports suggest affected female cases are more common; however more recently this view has been changed to one of equal prevalence in both genders. In my case, she is also a 28 years young female of Asian origin.

The most common signs and symptoms are lymphadenopathy, fever, sweats, malaise, anorexia, weight loss, hepatomegaly and leucopenia ${ }^{3}$. Viral aetiology of KFD is supported by its non-specific selfresolving symptoms, which are of slow, insidious onset. My case also has same presentation but there was no hepatomegaly.

A definite diagnosis is made by tissue biopsy, indeed whole lymph node biopsy. Histopathological assessment of affected lymph nodes reveals 
characteristic findings. There are three main patterns identified, proliferative, necrotizing and xanthomatous. The proliferative picture is seen in approximately a third of cases and has a dominant inflammatory infiltrate. Half of cases show necrotizing pattern and the xanthomatous type is rare and has abundant foam cells ${ }^{5}$. Immunoblast cell changes seen in lymph nodes mimic those of malignancy and are a source of diagnostic confusion. Cellular protein structures have been noted in the cytoplasm of lymphocytes and histiocytes that have also been found in those cells of patients with SLE. This adds strength to the hypothesis that KFD is a self-limiting SLE-like disorder.

As the symptoms are non-specific and some of the histological features are similar to other diseases it is easy to misdiagnose KFD with SLE or lymphoma. This is important as the treatment of KFD is symptomatic and supportive, spontaneous recovery is usual, while the latter two conditions require prompt specific treatments.

Long term follow-up of these patients is necessary as recurrent cases of KFD have been reported and there is some belief that KFD may be a precursor for SLE, as both diseases have had concurrent and co-existing disease patterns in the same patients ${ }^{6-8}$. In a review of KFD cases by Kucukardali et al the reported overall mortality rate associated with KFD is $2.1 \%$.

\section{Literature Review}

\section{History of KFD}

In 1972, Dr. Masahiro Kikuchi presented a case of lymphadenitis characterized by focal proliferation of reticular cells accompanied by numerous histiocytes and extensive nuclear debris in the Japanese Journal of the Haematological Society. In the same month, Dr. Fujimoto presented a similar case in a separate Japanese journal $^{10}$.

\section{Epidemiology}

KFD represents a condition most frequently found in East Asian and Japanese populations. The incidence is unknown but is rare in the UK and continental Europe. Extrapolating from the larger case series reports, the male to female ratio is roughly equal with slight female preponderance $^{11}$ and largely affects young adults $(<30$ years old).

\section{Aetiology}

The authors were unable to find any definitive evidence of an aetiological factor in the literature. Most articles on this subject concentrate on either an infective cause or an autoimmune disorder.

\subsection{Infection}

Yersinia enterocolitica, Brucellosis, Bartonella henselae, Entamoeba histolytica, Mycobacterium szulgai, and Toxoplasma gondii have been isolated in case reports. However, subsequent studies have failed to support these findings. The fact that most patients with KFD are unresponsive to antibiotics suggests that these microbiological organisms were incidental findings. Epstein-barr virus, herpes viruses, cytomegalo virus, parvovirus, paramyxo virus, parainfluenza virus, rubella, hepatitis B virus, Human Immunodeficiency Virus (HIV), human T-cell lymphotropic virus type 1, and the Dengue virus ${ }^{12-17}$ have all been implicated in the aetiology of KFD.

\subsection{Autoimmune}

Immunological screening of patients with KFD rarely reveals any autoimmune component in these patients. Imamura et $\mathrm{al}^{18}$ in 1982 performed an ultra-structural analysis of pathological tissue from patients with KFD, which drew some similarities with the histological findings in patients with systemic lupus erythematous (SLE). However, association of Kikuchi with SLE remains unclear.

There is also evidence of a genetic susceptibility for KFD. Tanaka et $\mathrm{al}^{19}$ found certain human leukocyte antigen (HLA) class II genes were more common found in those with KFD. These genes were found to be more common in Asian populations compared to Caucasian groups. The implication of this evidence is that KFD represents a self-limiting autoimmune response to an upper respiratory tract viral infection in geneticallysusceptible individuals. Some evidence suggests that KFD can initiate the onset of SLE, or is a short-lived version of SLE.

\section{Clinical Features}

The typical pattern of presentation is one of general flulike symptoms with posterior cervical, tender lymphadenopathy. Other less common nonspecific symptoms are headache, nausea, fatigue, and arthralgia. The disease process continues for approximately two to three months before resolving spontaneously ${ }^{20}$.

\section{Diagnosis}

The diagnosis of KFD is rather problematic due to its relative obscurity, nonspecific symptoms, and imprecise histological diagnosis. Currently, the only reliable method of diagnosis is histological examination of a lymph node excision biopsy. Unfortunately, samples from a fine-needle aspiration are generally not sensitive enough to provide a reliable diagnosis with an overall accuracy of $56.3 \%$. Blood tests classically show a mild neutropenia with mildly raised C-reactive protein (CRP) and erythrocyte sedimentation rate (ESR). 
Imaging techniques such as CT, MRI, PET/CT scanning also rarely provides conclusive diagnostic results $^{20,21}$. An incorrect provisional diagnosis of lymphoma or tuberculosis is made in many instances. In a study of 96 retrospective scans of confirmed KFD, Kwon et $\mathrm{al}^{22}$ found that on CT scanning, cases of KFD had the following typical appearances: (i) multiple homogeneous lymphadenopathy involving levels II to V, (ii) $94 \%$ were smaller than $2.5 \mathrm{~cm}$, this allows some differentiation from lymphoma which typically produces few but larger nodes, (iii) perinodal infiltration and necrosis is commonly found.

Ultrasonography scans frequently show lymph nodes with a hypoechoic center and a hyperechoic rim. Again, these features have a low specificity for KFD. These findings certainly do not allow the diagnosis of KFD with imaging techniques. However, linked to the clinical history, one may at least be suspicious of KFD rather than a neoplastic lesion.

A 2010 study by Kim et al. noted the following in cutaneous Kikuchi disease patients: (i) slight predominance of $\mathrm{CD} 8+$ lymphocytes, (ii) lymphohistiocytic infiltration and non-neutrophilic karyorrhexis (usually). The immunophenotype of Kikuchi disease is primarily composed of mature CD8positive and CD4-positive $\mathrm{T}$ lymphocytes. Lymphocytes and histiocytes also exhibit a high rate of apoptosis. There are relatively few B cells and natural killer (NK) cells are present. Positive immune-staining results by monoclonal antibody Ki-M1P are seen in Kikuchi disease but not in malignant lymphoma.

\section{Differential Diagnoses}

These are among (i) lymphoma, (ii) systemic lupus erythematous (SLE), (iii) infectious mononucleosis, (iv) kawasaki disease, (v) sarcoidosis, (vi) tuberculosis, (vii) syphilis.

\section{Management}

KFD is a self-limiting condition that rarely requires specific treatment in most cases. Management is, therefore, based on supportive therapy, such as analgesia and anti-inflammatory medication. In patients with neurological symptoms or in those cases where KFD is found with another medical condition, immunosuppression with corticosteroids appears to improve the patient's condition rapidly. A high initial oral dose of prednisolone with a subsequent reducing dose is the advocated regime. There are reports of excellent responses to hydroxychloroquine, immunoglobulins, and minocycline23-27. Recurrence of KFD is approximately $3 \%$.

\section{Conclusion:}

KFD is uncommon, but should include in a list of differential diagnoses of tender lymphadenopathy, especially affecting the cervical region. Its treatment differs significantly from the other conditions that would be on that list such as SLE, lymphoma and TB. Lymph node biopsy will aid accurate diagnosis, but if confusion with SLE occurs differentiation can be made with the aid of blood tests for complement levels amongst others. Recurrence has been reported. KFD has been reported within a family, but not between parent and child. It may suggest a genetic predisposition or a common environmental factor. Long term follow up will give us more data.

\section{References :}

1. Kikuchi M. Lymphadenitis showing focal reticulum cell hyperplasia with nuclear debris and phagocytosis. Nippon Ketsueki Gakkai Zasshi. 1972;35:378-80.

2. Fujimoto Y, Kozima Y, Yamaguchi K. Cervical subacute necrotizing lymphadenitis. A new clinicopathological agent. Naika. 1972;20:920-27.

3. Louis N, Hanley M, Davidson NM. Kikuchi-Fujimoto disease: a report of two cases and an overview. J Laryngol Otol. 1994;108:1001-4.

4. Altuntas F, Canoz O, Yildiz O, Eser B, Cetin M, Unal A. KikuchiFujimoto disease: a rare but important cause of fever and lymphadenopathy in pregnant women. Am J Haematol. 2006;81:118-20.

5. Kuo TT. Kikuchi's disease (histiocytic necrotizing lymphadenitis), a clinico-pathologic study of 79 cases with an analysis of histiolytic subtypes, immunohistology and DNA ploidy. Am J Surg Pathol. 1995:19(7):798-809.

6. Santana A, Lessa B, Galrao L, Lima I, Santiago M. KikuchiFujimoto's disease associated with systemic lupus erythematous: case report and review of literature. Clin Rheumatol. 2005;24:60-63.

7. Famularo G, Giustiniani MC, Marasco A, Minisola G, Nicotra GC, De Simone C. Kikuchi-Fujimoto lymphadenitis: case report and literature review. Am J Haematol. 2003;74:60-63.

8. Lin HC, Su CY, Huang CC. Kikuchi's disease: a review and analysis of 61 cases. Otolaryngol Head Neck Surg. 2003;128:650-53.

9. Kucukardali Y, Solmazgul E, Kunter E, Oncul O, Yildirim S, Kaplan M. Kikuchi-Fujimoto Disease: analysis of 244 cases. Clin Rheumatol. 2007;26:50-54.

10. Fujimoto Y, Kojima Y, Yamaguchi K. "Cervical subacute necrotizing lymphadenitis," A New Clinicopathological Entity. Naika 1972; 20: 920-27.

11. Lin HC, Su CY, Huang CC, Hwang CF, Chien CY. "Kikuchi's disease: a review and analysis of 61 cases," Otolaryngology 2003;128(5): 650-53.

12. Heikens J, Tel W, van de Stadt J, de Koning J, Napel CHHT. "Kikuchi's lymphadenitis: report of a Yersinia enterocoliticaassociated case and an overview of aetiology and clinical outcome," Netherlands Journal of Medicine 1992; 41(5):222-28. 
13. Charalabopoulos K, Papalimneou V, Charalabopoulos A, Bai M, Agnantis N. "Brucella melitensis infection stimulates an immune response leading to Kikuchi-Fujimoto disease". In Vivo 2003; 17(1):51-53.

14. Chung JY, Sang WK, Tae HH, Sung JL. "Detection of the Bartonella henselae gene sequence in lymph nodes of children with Kikuchi's disease," Pediatrics 2005; 115(4): 1112.

15. Aydogan T, Kanbay M, Uraldi C, Kaya A, Uz B, Isik A, et al. "Kikuchi Fujimoto disease secondary to entamoeba histolytica: case report," Journal of Infection 2006;53(4):171-e173.

16. Maek-a-nantawat W, Viriyavejakul P. "Mycobacterium szulgai lymphadenitis mimicking Kikuchi's disease in Thailand," Southeast Asian Journal of Tropical Medicine and Public Health 2001;32(3):537-40.

17. Kikuchi M, Yoshizumi T, Nakamura H. "Necrotizing lymphadenitis: possible acute toxoplasmic infection". Virchows Archiv. 1977;376(3):247-53.

18. Imamura M, Ueno H, Matsuura A, Kamiya H, Suzuki T, Kikuchi $\mathrm{K}$, et al. "An ultrastructural study of subacute necrotizing lymphadenitis," American Journal of Pathology 1982;107(3):29299.

19. Tanaka T, Ohmori M, Yasunaga S, Ohshima K, Kikuchi M, Sasazuki T. "DNA typing of HLA class II genes (HLA-DR, -DQ and -DP) in Japanese patients with histiocytic necrotizing lymphadentis (Kikuchi's disease)". Tissue Antigens 1999;54(3):246-53.

20. Khanna D, Shrivastava A, Malur PR, Kangle R. "Necrotizing lymphadenitis in systemic lupus erythematosus: is it KikuchiFujimoto disease?" Journal of Clinical Rheumatology2010;16(3):123-24.

21. Tong TR, Chan OW, Lee KC. "Diagnosing Kikuchi disease on fine needle aspiration biopsy: a retrospective study of 44 cases diagnosed by cytology and 8 by histopathology". Acta Cytologica 2001;45(6):953-57.

22. Ito K, Morooka M, Kubota K. "Kikuchi disease: 18F-FDG positron emission tomography/computed tomography of lymph node uptake". Japanese Journal of Radiology2010;28(1):15-19.

23. Na DG, Chung TS, Byun HS, Kim HD, Ko YH, Yoon JH. "Kikuchi disease: CT and MR findings". American Journal of Neuroradiology 1997;18(9):1729-32.

24. Douglas M, Bradbury R, Kannangara S, Mitchell D. "Arthritis as an unusual manifestation of Kikuchi-Fujimoto disease". Rheumatology 2003; 42(8): 1010-12.

25. Jang YJ, Park KH, Seok HJ. "Management of Kikuchi's disease using glucocorticoid". Journal of Laryngology and Otology 2000;114(9): 709-11.

26. Mahmood A, Mir R, Salama SR, Miarrostami RM, Lapidus C, Pujol F. "Kikuchi's disease: an unusual presentation and a therapeutic challenge". Yale Journal of Biology and Medicine 2006; 79(1):27-33.

27. Rezai K, Kuchipudi S, Chundi V, Ariga R, Loew J, Sha BE. "Kikuchi-Fujimoto disease: hydroxychloroquine as a treatment". Clinical Infectious Diseases 2004; 39(12): e124-e126. 